\title{
MEJORAMIENTO DE LA PRODUCTIVIDAD EN EAF*
}

Mario Andres Albuja Ribadeneira ${ }^{1}$

\section{Resumen}

El presente trabajo pretende encontrar la ruta para incrementar la productividad y reducir el costo de transformación en una acería eléctrica, mediante el uso de la tecnología disponible en el mercado. El método para alcanzar los resultados se enfocan en una drástica reducción de los tiempos de transformador conectado (power-on), los tiempos de transformador apagado (power-off) asi como la reducción en los consumos de las materias primas e insumos para la fabricación de acero por la ruta del horno eléctrico. La experiencia de Adelca arroja resultados que sobrepasan el $100 \%$ de productividad y $30 \%$ de reducción en el costo de transformación de la palanquilla. Los resultados obtenidos sin duda alguna servirán de una guía probada para quienes buscan un mejor desempeño en el negocio del acero.

Palabras-clave: Productividad en horno eléctrico; Reducción power-on; Reducción power-off.

\section{Abstract}

\section{EAF PRODUCTIVITY IMPROVEMENT}

This paper pretend to give the route to the productivity improvement and transformation reduction cost in the EAF meltshop, using the the best available technology.The way to reach the outputs are focused in the power-on and power-off drastic reduction, as well as lower raw materials and consumables comsumption. The outputs obteined by Adelca related with the productivity exceed the $100 \%$ with the transformation cost reduction around of $50 \%$. The Adelca success will be undoubtedly an important help to people who are thinking to improve the meltshop performance.

Keywords: Productivity improvement. 


\section{INTRODUCCIÓN}

\subsection{Objetivos}

El presente trabajo tiene como principal objetivo demostrar el dramático impacto sobre la productividad de Horno eléctrico cuando se utiliza un alto porcentaje de chatarra Shredder en el Mix de carga. Es conocido que en la actualidad el uso de $40 \%-50 \%$ de este tipo de chatarra genera un alto desempeño del Horno eléctrico, sin causar graves problemas operacionales, pero el uso de $80 \%$ - $90 \%$ si representa un verdadero reto, ya que sobrepasando los problemas operacionales que esta practica puede causar, los resultados del EAF son excepcionales; resultados que solo son factibles mediante la aplicación de una solución integral que consiste en la preparación de la chatarra Shredder, el uso de un transformador lo suficientemente potente con una relación de al menos 1,25 veces MVA versus carga de chatarra y la implementación de un sistema de recuperación de metales ferrosos de los residuos obtenidos del procesamiento de la chatarra en la máquina Shredder. La implementación de esta solución integral ha generado interesantes conclusiones la la compañía, las cuales van a ser expuestas en el presente trabajo.

\subsection{Antecedentes}

Adelca es la mayor y mas importante empresa siderúrgica del Ecuador, ubicada a poca distancia de la ciudad de Quito y tiene una capacidad instalada de 330.000 tpa de acero liquido y similar cantidad en productos laminados como barra de refuerzo y perfiles. Adicionalmente tiene una planta de trefilacion para la fabricación de productos de alambre con una capacidad de 60.000 tpa.

En el año 2006 Adelca contrato con la empresa Siderengineering (Udine-Italia), la construcción de una planta de Acería (EAF-LF-MCC) con una capacidad de hasta 150.000 tpa. Dicha planta inicio operaciones en el mes de julio del 2008.

Al igual que la mayoría de los nuevos participantes en la industria de fabricación de acero, la empresa comenzó sus operaciones enfocada en el control del proceso y sus costos, especialmente en lo relacionado a consumos energéticos, mano de obra y consumibles, pero con muy poco interés en el factor, que mas adelante se convertiría en el de mayor impacto en su desempeño: La chatarra

\subsection{La Chatarra en el Ecuador}

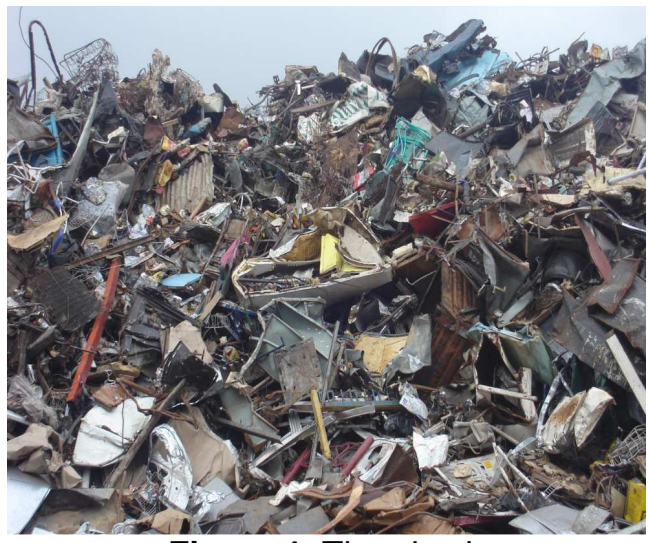

Figura 1. Tipo de chatarra

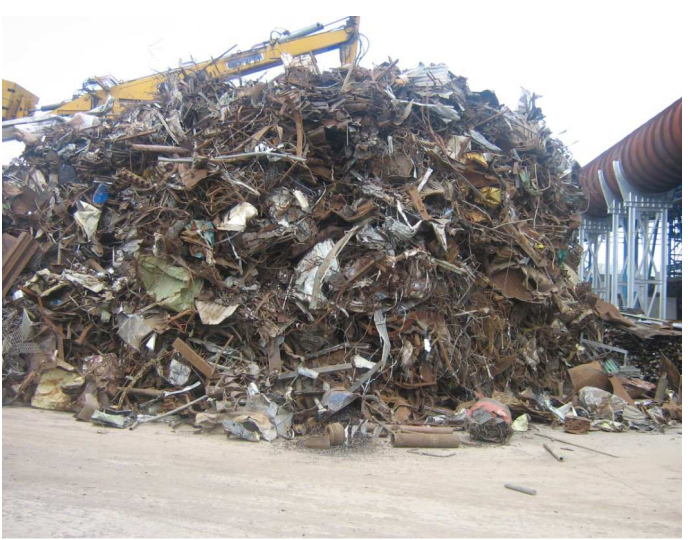

Figura 2. Tipo de chatarra 


\subsection{Mix Original de Carga: Chatarra Prensada-Cortada y Pacas}

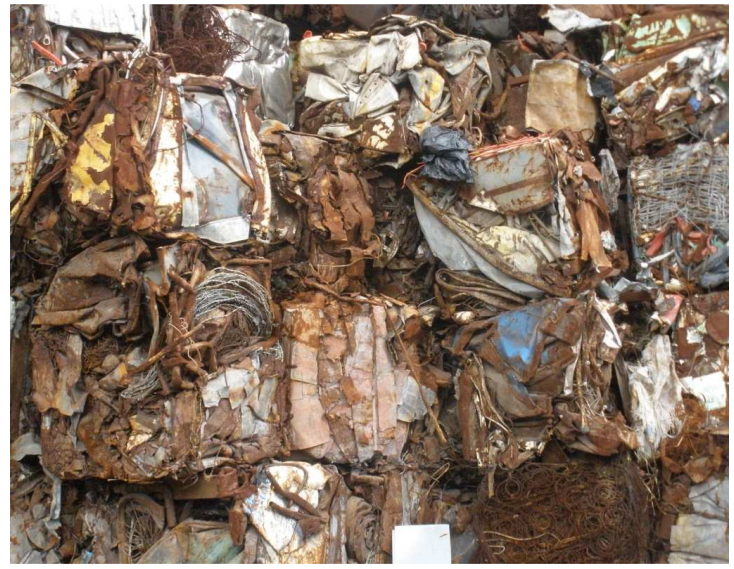

Figura 3. Chatarra Pacas.

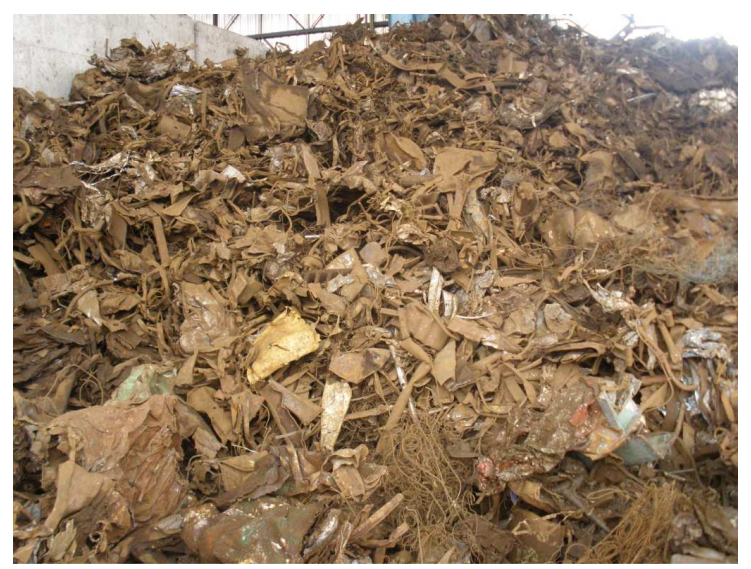

Figura 4. Chatarra Prensada-Cortada.

\subsection{Material No-Ferroso e la Chatarra: Tierra, Piedras, Plásticos, Electrónicos y Otros}

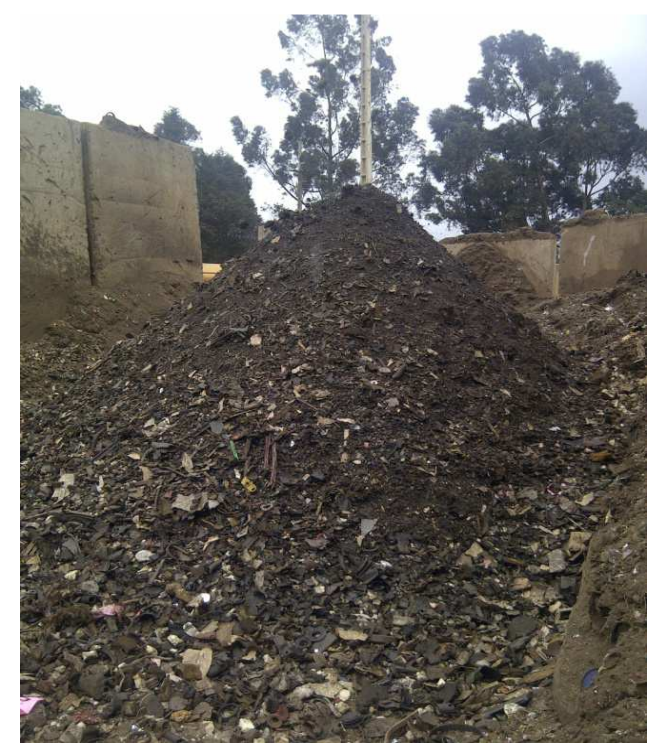

Figura 5. Tierra extraida de la chatarra.

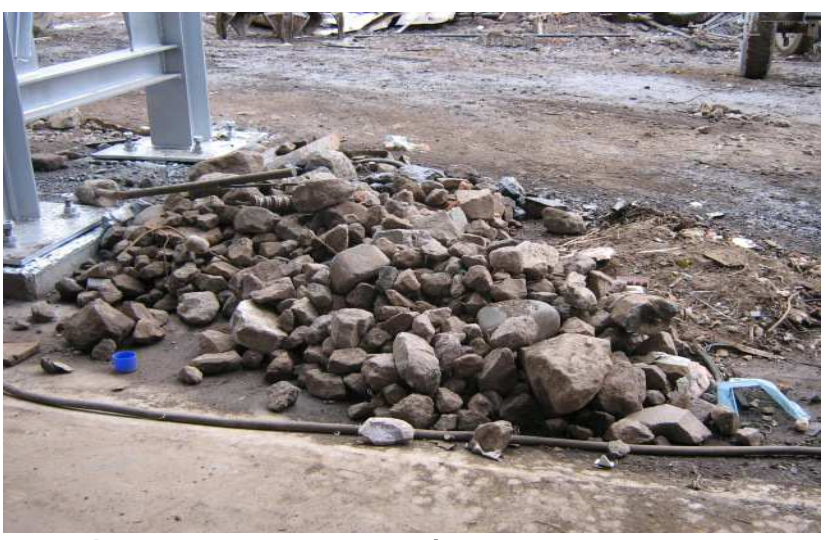

Figura 6. Piedras extraídas de la chatarra.

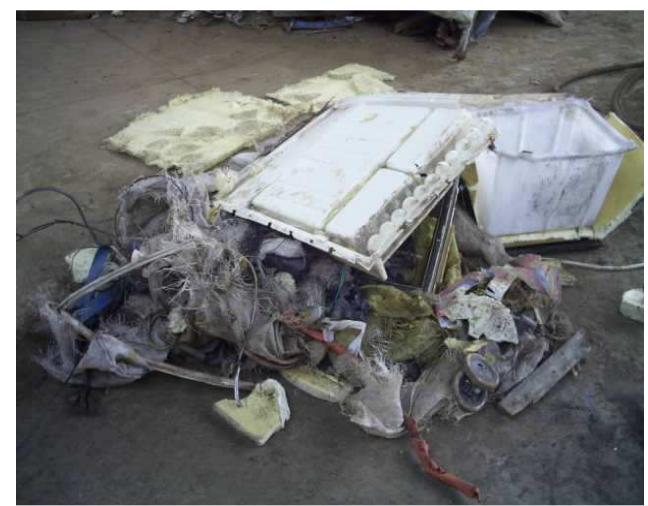

Figura 7. Plasticos y elctronicos 
Tabla 1. Mix de carga original

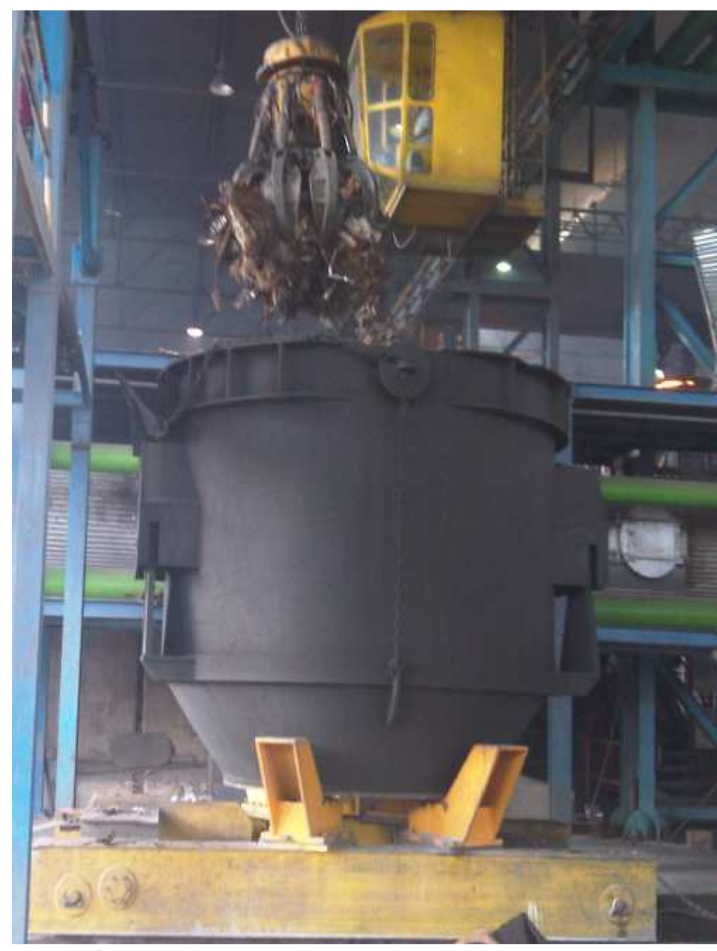

\begin{tabular}{|c|c|}
\hline Prensado-cortado & $50 \%$ \\
\hline Pacas & $30 \%$ \\
\hline Oxicorte & $10 \%$ \\
\hline Hierro fundido & $5 \%$ \\
\hline Otros & $5 \%$ \\
\hline
\end{tabular}

Figura 8. Proceso de carga de cesta

Tabla 2. Características de Mix de carga original

\begin{tabular}{|c|c|c|}
\hline Densidad de la carga & 0,45 & ton $/ \mathrm{m}^{3}$ \\
\hline Chatarra ferrosa & $82 \%-85 \%$ & \\
\hline Tierra $\left(65 \%-75 \% \mathrm{SiO}_{2} \& \mathrm{Al}_{2} \mathrm{O}_{3}\right)$ & $10 \%-12 \%$ & \\
\hline Plasticos-PVC-Otros & $4 \%-5 \%$ & \\
\hline Chatarra-no ferrosa & $2 \%-3 \%$ & \\
\hline
\end{tabular}

Características generales de la carga:

- Baja densidad

- Poca pureza (suciedad)

- Tiempos de carga y fusión altos 


\subsection{Estadísticas Operacionales Originales}

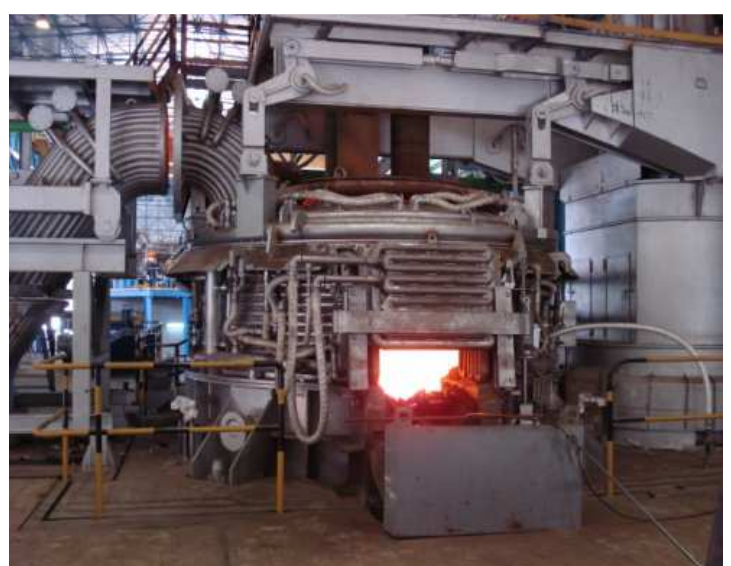

Figura 9. Horno electrico.
Tabla 3. Resultados orginales

\begin{tabular}{|l|l|l|}
\hline Carga de chatarra & 32,0 & ton \\
\hline Numero de cestas & 5,0 & \\
\hline Cales & 62,0 & $\mathrm{~kg} /$ ton \\
\hline Carbon & 21,9 & $\mathrm{~kg} /$ ton \\
\hline Ferroaleaciones & 15,1 & $\mathrm{~kg} /$ ton \\
\hline Oxigeno & 36,0 & $\mathrm{Nm}$ '/ton \\
\hline LPG (Liquid Petroleum Gas) & 4,0 & $\mathrm{Nm}$ /ton \\
\hline Consumo de elctrodos & 2,8 & $\mathrm{~kg} /$ ton \\
\hline Consumo de refractarios & 18,0 & $\mathrm{~kg} /$ ton \\
\hline Rendimiento metalico & $85 \%$ & \\
\hline Vaciado & 27,2 & ton \\
\hline o C Temperatura de vaciado & 1615,0 & -C \\
\hline Consumo de energia & 580,0 & $\mathrm{kwh} /$ ton \\
\hline Power on & 50,2 & $\mathrm{minutos}$ \\
\hline Power off & 17,5 & $\mathrm{minutos}$ \\
\hline Tap to tap & 67,7 & $\mathrm{minutos}$ \\
\hline Transformador & 25,5 & $\mathrm{MVA}$ \\
\hline Potencia activa promedio & 18,9 & $\mathrm{MW}$ \\
\hline Mantenimiento y operacion & $19 \%$ & \\
\hline Tiempo real de fundicion & $\mathbf{8 1} \%$ & \\
\hline Productividad & 19,54 & ton/hr \\
\hline Producción mensual & 14,067 & ton/mes \\
\hline Escoria & $20 \%$ & \\
\hline
\end{tabular}

\section{EL PROYECTO}

En febrero 2010 adelca decide cambiar el transformador del EAF pasando de 25 MVA a 42 MVA, el contrato fue asignado a la empresa WEG, Division-Brazil, dos meses mas tarde firma un contrato con The Shredder Coompany, Texas-USA, para la adquisición de un una máquina Shredder-4000 HP. Adelca tenia la firme convicción que estas dos decisiones aportarían sustancialmente a la mejora de sus operaciones, pues algunos meses atrás había probado el uso de material procesado Shredder hasta en un $50 \%$ del Mix de carga con total éxito. En abril de 2011, se comenzó la operación con estos nuevos equipamientos y tres meses más tarde los resultados planificados fueron obtenidos. 


\subsection{Nuevo Mix de Carga}

Tabla 4. Nuevo Mix de carga

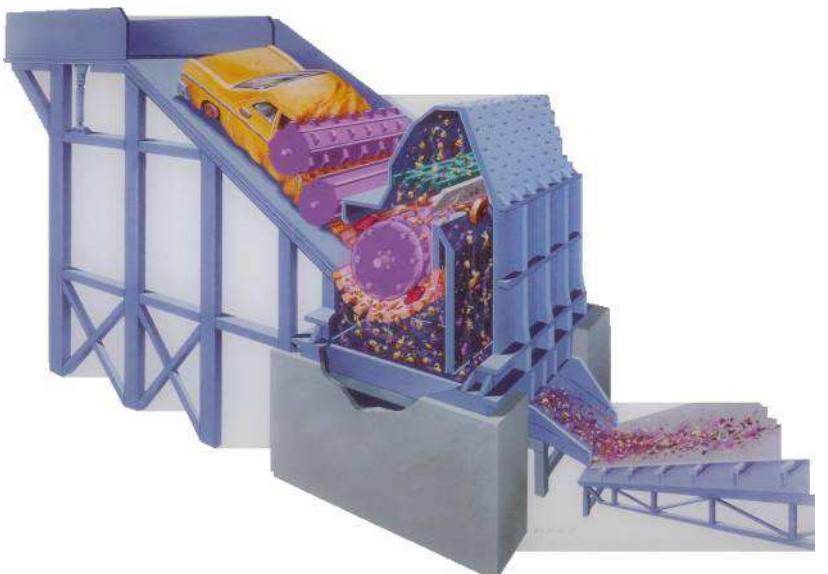

\begin{tabular}{|c|c|}
\hline Chatarra Shredder & $85 \%$ \\
\hline Pacas & $4 \%$ \\
\hline Oxicorte & $8 \%$ \\
\hline Hierro fundido & $2 \%$ \\
\hline Otros & $1 \%$ \\
\hline
\end{tabular}

Figura 10. Esquema de equipo Shredder.

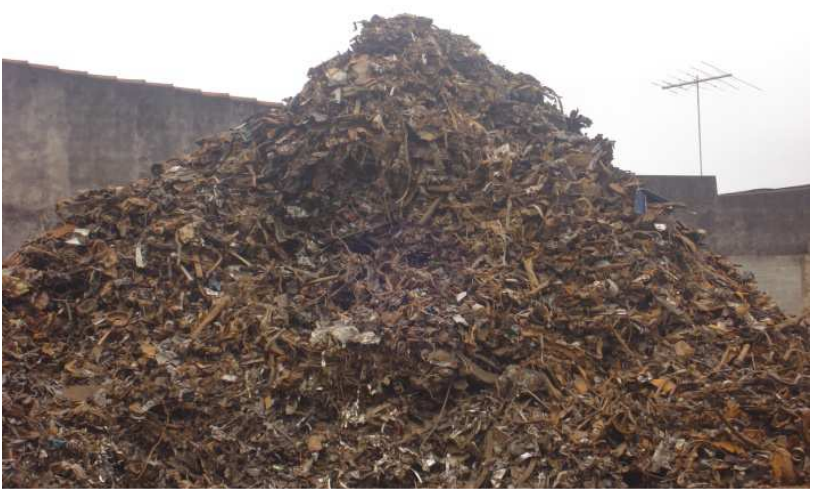

Figura 11. Chatarra Shredder

Tabla 5. Caracteristicas de nuevo Mix de carga

\begin{tabular}{|c|c|c|}
\hline Nueva densidad & 0,9 & ton $/ \mathrm{m}^{3}$ \\
\hline Chatarra ferrosa & $95 \%-96 \%$ & \\
\hline Tierra $\left(65 \%-75 \% \mathrm{SiO}_{2} \& \mathrm{Al}_{2} \mathrm{O}_{3}\right)$ & $2 \%-3 \%$ & \\
\hline Plásticos-PVC-Otros & $1 \%-2 \%$ & \\
\hline Chatarra no-ferrosa & $\approx 0 \%$ & \\
\hline
\end{tabular}




\subsection{Resultados de la Nueva Operación}

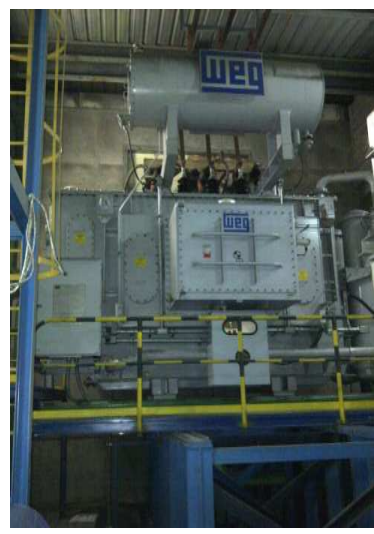

Figura 12. Transformador

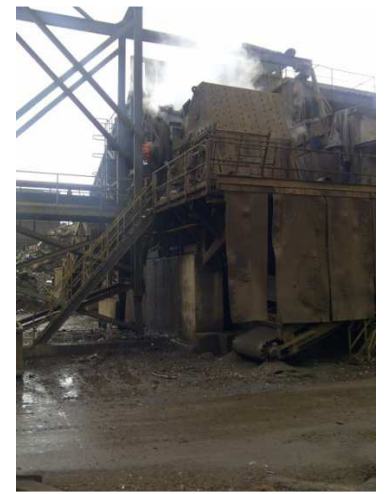

Figura 13. Equipo Shredder

Tabla 6. Comparación de los resultados originales y los resultados con nuevo Mix de carga

\begin{tabular}{|c|c|c|c|}
\hline Carga de Chatarra & 32,0 & 32,0 & Ton \\
\hline Numero de Cestas & 5,0 & 2,0 & \\
\hline Cales & 62,0 & 29,4 & $\mathrm{~kg} / \mathrm{ton}$ \\
\hline Carbon & 21,9 & 21,9 & $\mathrm{~kg} / \mathrm{ton}$ \\
\hline Ferroaleaciones & 15,1 & 15,1 & $\mathrm{~kg} / \mathrm{ton}$ \\
\hline Oxigeno & 36,0 & 36,0 & $\mathrm{Nm}^{3} /$ ton \\
\hline LPG (Liquid Petroleum Gas) & 4,0 & 4,0 & $\mathrm{Nm}^{3} /$ ton \\
\hline Consumo de electrodos & 2,8 & 1,6 & $\mathrm{~kg} / \mathrm{ton}$ \\
\hline Consumo de refractario & 18,0 & 7 & $\mathrm{~kg} / \mathrm{ton}$ \\
\hline Rendimiento metalico & $85 \%$ & $93 \%$ & \\
\hline Vaciado & 27,2 & 30 & ton \\
\hline o C Temperatura de vaciado & 1615,0 & 1625,0 & 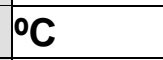 \\
\hline Consumo de energia & 580,0 & 440,0 & kwh/ton \\
\hline Power on & 50,2 & 29,0 & minutos \\
\hline Power off & 17,5 & 9,0 & minutos \\
\hline Tap to tap & 67,7 & 38,0 & minutos \\
\hline Transformador & 25,5 & 42,0 & MVA \\
\hline Potencia activa & 18,9 & 29 & MW \\
\hline Mantenimiento y operacion & $19 \%$ & $12 \%$ & \\
\hline Tiempo total de fundicion & $81 \%$ & $88 \%$ & \\
\hline Productividad & 19,54 & 40,0 & ton $/ \mathrm{h}$ \\
\hline Produccion mensual & 14.000 & 30.000 & ton/mes \\
\hline Escoria & $20 \%$ & $9 \%$ & \\
\hline
\end{tabular}

\subsection{Operación Metales No-Ferrosos}

En Octubre 2013, Adelca puso en operación una planta de recuperación de metales no-ferrosos cuya recuperación de la inversión fue casi inmediata. Actualmente exporta a la china una interesante cantidad de metales no-ferrosos tales como zorba, zurich, twich y acero inoxidable; todo el material es recuperado de los residuos obtenidos del procesamiento de la chatarra en la máquina Shredder. 


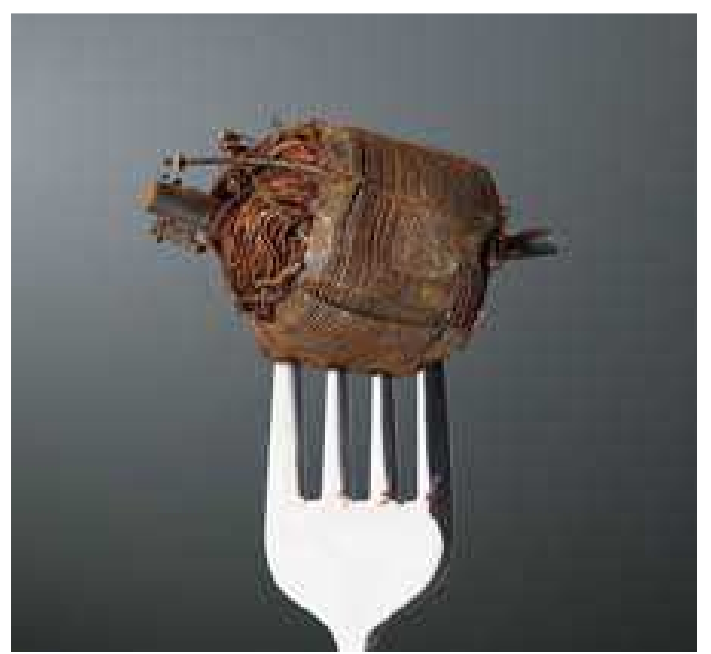

Figura 13. No ferroso Cobre "meatball"

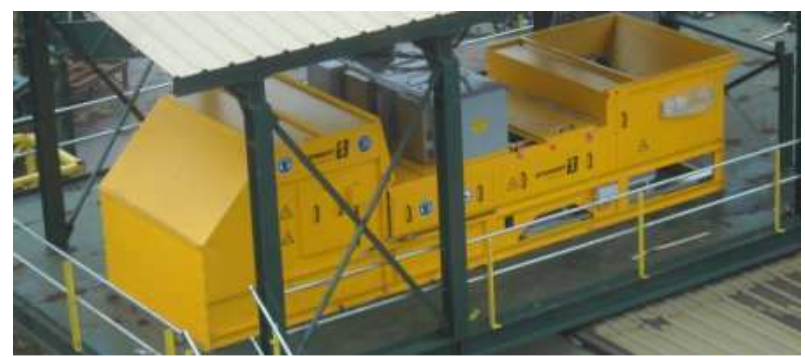

Figura 14. Separador de no ferrosos Eddy- current

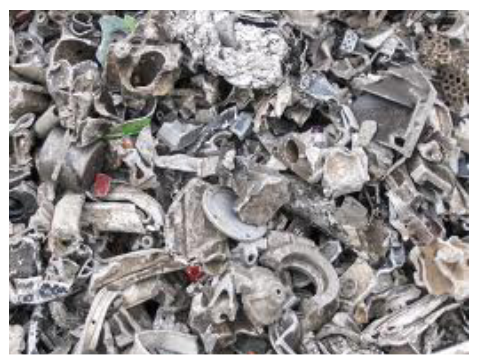

Figura 15. Chatarra de Aluminio

\section{COCLUSIONES}

El proceso de preparación de chatarra mediante el equipo Shredder tiene un poderoso impacto en la industria del acero via EAF, el incremento de productividad por esta razon supera el $100 \%$ y la reducción de costo atribuible a la misma es aproximadamente $30 \%$, lo que representa entre $35 \$ / t-40 \$ / t$, considerando los preciso de mano de obra y materias primas en Ecuador; la productividad se incrementa proporcionalmente al \% de uso de la chatarra Shredder, doble uso, doble resultado en productividad.

El aumento de la potencia del transformador es estratégico en si mismo, mejora radicalmente el tiempo de fusion power-on, e incrementa la capacidad para fundir altos porcentajes de chatarra Shredder, impidiendo que la esta forme grandes masas en las paredes del horno, lo cual genera problemas operacionales que desembocan en extensas paradas del proceso; es la unica herramienta poderosa que permite fundir hasta un $90 \%$ de chatarra Shredder en el mix de carga.

El valor del metal no-ferroso recuperado de los residuos del proceso Shredder reduce entre $25 \$ / t$ y $30 \$ / t$ el costo de la chatarra. 